\title{
2003-2004 NONLINEAR OPTICS MEASUREMENTS AND MODELING FOR THE CERN SPS
}

\author{
G. Arduini, F. Zimmermann, CERN, Geneva, Switzerland; \\ A. Faus-Golfe,IFIC-Valencia, Spain; R. Tomas, CELLS, Spain
}

\section{Abstract}

In 2003 and 2004 nonlinear chromaticity, amplitude detuning, chromatic phase advance, resonance driving terms and off-energy orbits were measured in the CERN SPS at $14 \mathrm{GeV} / \mathrm{c}$ and $26 \mathrm{GeV} / \mathrm{c}$, respectively. From the nonlinear chromaticity, the SPS optics model has been updated, by adjusting the strength of nonlinear field errors in dipoles and quadrupoles. Furthermore, we have added to the model the effect of the displacement of all main bends and the voluntary misalignments of all the other elements of the machine. We compare the field errors with those founded in 2002, 2001 and 2000. The tune shifts with transverse amplitude, driving terms, etc., predicted by this nonlinear optics model are compared with direct measurements.

\section{INTRODUCTION}

Since 2000 we are developing procedures by which the SPS nonlinear optics model can be updated from a fast measurement of the nonlinear chromaticity. This procedure is not only of interest for the SPS, which as LHC injector must provide high-quality beams, but it also allows us to explore experimental techniques for commissioning of the LHC itself. Previous studies of 2000 and 2001 were reported in [1], the 2002 experiments and their analysis in [2], and an application to the full SPS cycle in [3]. The quality of the optics model can be verified by comparing model predictions and complementary measurements.

In this paper, we describe the results of two optics studies conducted in 2003 and 2004. Specifically, we measured linear and nonlinear chromaticity, detuning with amplitude, chromatic $\beta$ functions, central orbit, dispersion, and resonant driving terms. The 2003 measurement was performed at a momentum of $14 \mathrm{GeV} / \mathrm{c}$, the 2004 one at $26 \mathrm{GeV} / \mathrm{c}$, i.e., at the same momentum as all the previous measurements, but this time for the real SPS cycle for LHC. Remanent fields are expected to depend both on the beam energy and on the cycle, and, therefore, the optics models obtained for 2003 or 2004 are expected to differ from the earlier ones.

In addition, the SPS optics layout from which the nonlinear model is constructed was improved for the present analysis by introducing the voluntary misalignments of various machine elements. Namely, the main dipole magnets of the SPS are displaced by $4.8 \mathrm{~mm}$ in the radially outward direction to maximize the physical aperture including the sagitta. Three quadrupoles in the injection region are displaced to create the dogleg for injection and beam dump and eight dipoles upstream of the extraction regions in straight sections 2 and 6 are displaced to increase the aperture for the beam when bumped for the slow extraction. Also, in 2004 a few of the sextupoles were moved to new locations in order to increase the dynamic aperture [4], and their new positions are included in the 2004 model.

Our analysis is applied to both data from a single pickup ("tune meter"), and to the turn-by-turn readings of the beam-position monitors (BPMs). Using the latter, we can achieve a higher resolution and also obtain additional information on the local optics [2].

\section{NONLINEAR CHROMATICITY}

Figure 1 presents the two betatron tunes as a function of the momentum offset, measured by the tune meter and by all BPMs, for the experiment in $2003(14 \mathrm{GeV} / \mathrm{c})$ and 2004 (LHC cycle, $26 \mathrm{GeV} / \mathrm{c}$ ). Superimposed on the data are fits of the linear and nonlinear chromaticity through $3 \mathrm{rd}$ order in the relative momentum deviation $\delta$, and also the reproduction by the nonlinear model which is constructed by matching multipole errors to the fitted chromaticity curves (see later). The fitted linear and nonlinear chromaticity components are summarized in Table 1.
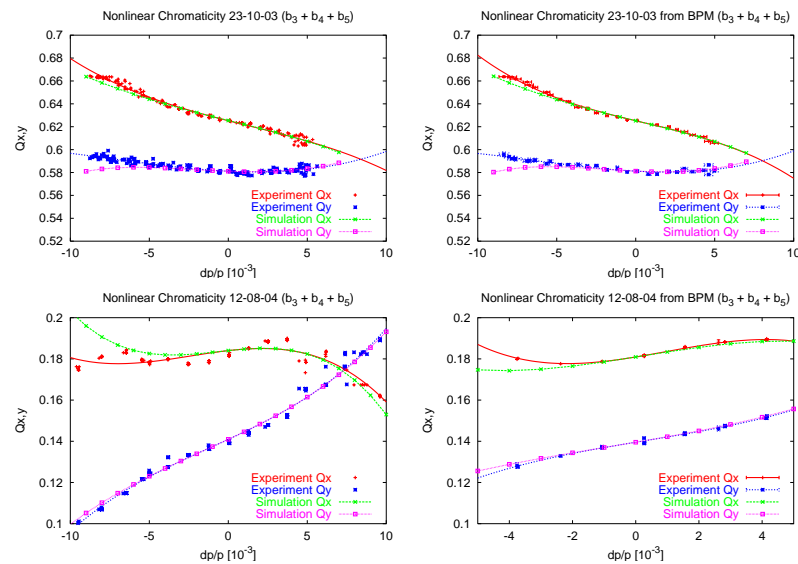

Figure 1: Measured, fitted and predicted horizontal and vertical tune versus momentum offset, for the experiment on $23^{\text {rd }}$ October 2003 (top) and on $12^{\text {th }}$ August 2004 (bottom); all data were fitted over a momentum range $\delta$ from -0.01 to 0.01 ; the data on the left plots are from a dedicated application ('tune meter'), the right ones were obtained by averaging over all BPMs.

The procedure for matching the linear and nonlinear chromatic coefficients for each of the experiments using MAD [5] is described in detail in [1] and [2]. By this matching, we are determining systematic sextupole and decapole errors in two families of dipole magnets as well as octupole components in the focusing and defocusing quadrupoles, so as to best reproduce the measured nonlinear chromaticity. In the present case, due to the intro- 


\begin{tabular}{|l|r|r|r|r|}
\hline MD's & $Q_{0 x}$ & $Q_{x}^{\prime}$ & $1 / 2 Q_{x}^{\prime \prime}$ & $1 / 6 Q_{x}^{\prime \prime \prime}$ \\
$\mp d p / p$ fit & $Q_{0 y}$ & $Q_{y}^{\prime}$ & $\begin{array}{r}1 / 2 Q_{y}^{\prime \prime} \\
1 / 6 Q_{y}^{\prime \prime \prime} \\
{\left[10^{2}\right]}\end{array}$ & {$\left[10^{4}\right]$} \\
\hline \hline $23 / 10 / 2003$ & 0.625 & -3.35 & 0.29 & -2.00 \\
& 0.581 & -0.57 & 1.66 & 0.49 \\
\hline $23 / 10 / 2003$ & 0.625 & -3.30 & 0.35 & -2.08 \\
$(\mathrm{BPMs})$ & 0.581 & -0.74 & 1.66 & 0.85 \\
\hline \hline $12 / 08 / 2004$ & 0.184 & 0.87 & -1.41 & -1.95 \\
& 0.141 & 3.52 & 0.46 & 1.40 \\
\hline $12 / 08 / 2004$ & 0.180 & 2.52 & 2.76 & -9.44 \\
(BPMs) & 0.140 & 2.53 & -0.33 & 3.09 \\
\hline
\end{tabular}

Table 1: Linear and nonlinear chromaticity components obtained from a polynomial fit of the measured betatron tunes as a function of $r$ frequency.

duction of the displacements, which give rise to feeddown effects, we found it necessary to rematch simultaneously at each step the tunes at $\delta=0$ with the main quadrupoles families and the tunes at $\delta \neq 0$ using all the other multipoles. The fit range in $\delta$ was chosen from -0.005 to 0.005 , since this was found to be optimum in [2]. The fitted multipole components are compiled in Table 2. The multipole errors found at $14 \mathrm{GeV} / \mathrm{c}$ show little similarity with the 26 $\mathrm{GeV} / \mathrm{c}$ values; in several cases even the sign is different. This type of behavior was expected from historical magnet measurements [6]. The predictions from the nonlinear optics models so obtained are indicated in Fig. 1, together with the measured and fitted chromaticity curves.

We note that an octupole component is not natural for the quadrupole geometry. It is possible, that the small octupolar term visible in the chromaticity only arises as a feeddown from the dipole decapole component due to the magnet sagitta.

\section{DETUNING WITH AMPLITUDE}

The tune shifts with amplitude were measured in both experiments, after adjusting the Landau-damping octupoles so that their values were close to zero. The beam was kicked to various amplitudes in either one or both planes and the corresponding tunes were recorded. The detuning with amplitude was also simulated using the SIXTRACK code [7].

\section{ORBIT AND DISPERSION}

Plotting the standard deviation of the horizontal orbit as a function of the average orbit, while varying the beam energy, reveals a minimum at $\delta \approx 0$, which can be used to determine the "central orbit" of the storage ring. Figure 2 shows this curve for the BPM measurements in 2004. The slopes of the aymptotes on either side of the minimum correspond to the ratio of $\sigma_{D} /\langle D\rangle$, where $\sigma_{D}$ is the standard deviation of the dispersion function and $\langle D\rangle$ is the average dispersion function.

The dispersion was measured at every BPM by performing a fit of the closed orbit versus $\delta$. Horizontal and ver-

\begin{tabular}{|l|r|r|r|}
\hline element & dipoles & quadr. & dipoles \\
\hline MDs & $b_{3 a}$ & $b_{4 f}$ & $b_{5 a}$ \\
$\mp d p / p$ fit & $b_{3 b}$ & $b_{4 d}$ & $b_{5 b}$ \\
\hline units & $10^{-3}\left[m^{-2}\right]$ & $10^{-1}\left[m^{-3}\right]$ & {$\left[m^{-4}\right]$} \\
\hline \hline $18 / 10 / \mathbf{2 0 0 0}$ & 1.366 & 0.808 & -5.833 \\
$(26 \mathrm{GeV} / \mathrm{c})$ & -0.826 & -2.551 & -5.833 \\
\hline \hline average 2001 & 1.152 & 1.059 & -19.799 \\
$(26 \mathrm{GeV} / \mathrm{c})$ & -2.968 & -0.768 & -19.799 \\
\hline \hline average 2002 & 0.621 & -0.206 & -11.831 \\
$(26 \mathrm{GeV} / \mathrm{c})$ & -2.541 & -1.486 & -11.831 \\
\hline \hline $23 / 10 / \mathbf{2 0 0 3}$ & -0.933 & -1.463 & 1.075 \\
$(14 \mathrm{GeV} / \mathrm{c})$ & 0.707 & 5.579 & -8.624 \\
\hline $23 / 10 / \mathbf{2 0 0 3}$ & -0.940 & -1.457 & 1.187 \\
$(\mathrm{BPMs})$ & 0.754 & 5.577 & -10.298 \\
\hline \hline $12 / 08 / \mathbf{2 0 0 4}$ & 0.561 & -0.199 & -3.892 \\
$(26 \mathrm{GeV} / \mathrm{c})$ & -1.190 & 0.007 & 0.958 \\
\hline $12 / 08 / \mathbf{2 0 0 4}$ & 0.855 & 0.047 & -4.728 \\
$(\mathrm{BPMs})$ & -1.109 & -0.002 & 0.033 \\
\hline
\end{tabular}

Table 2: Multipole components of dipoles and quadrupoles obtained by MAD matching in 2003 and 2004 compared with earlier results $[1,2]$. Differences between the new and old results are attributed to the different beam energy or cycle, respectively. If the same value is quoted for $b_{5 a}$ and $b_{5 b}$, a common decapole error was assumed for the two kinds of dipoles.

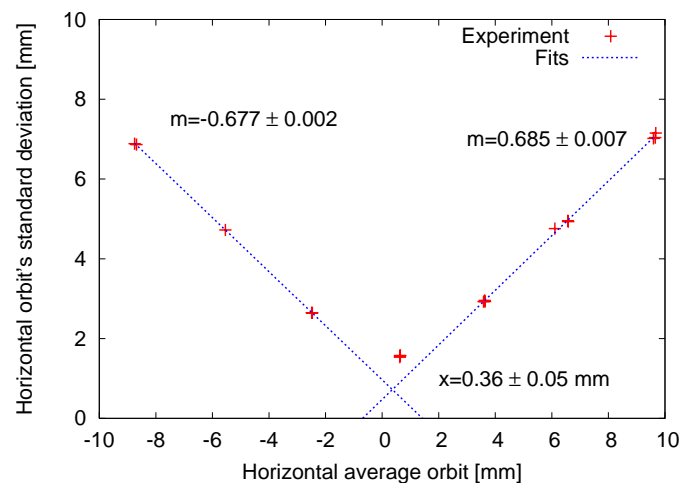

Figure 2: Standard deviation of the horizontal orbit vs. the average horizontal orbit.

tical dispersion functions for 2003 are compared with the model in Fig. 3. The agreement is excellent in the horizontal plane. The spurious vertical dispersion is only about $1 \%$ of the horizontal, and it can be reproduced by the model after fitting the strength of 4 vertical correctors (MDV10107, MDV22307, MDV43107, MDV60707 with fitted deflection angles $-10,30,-30$ and $-10 \mu \mathrm{rad}$, respectively).

\section{CHROMATIC BETA FUNCTION}

The beta function varies with the beam energy, to first order, as $\beta=\beta_{0}+\beta^{\prime} \delta$, where $\delta$ is the relative energy deviation, and $\beta^{\prime}$ denotes the derivative with respect to $\delta$. To become independent of the BPM calibration, we compute the 

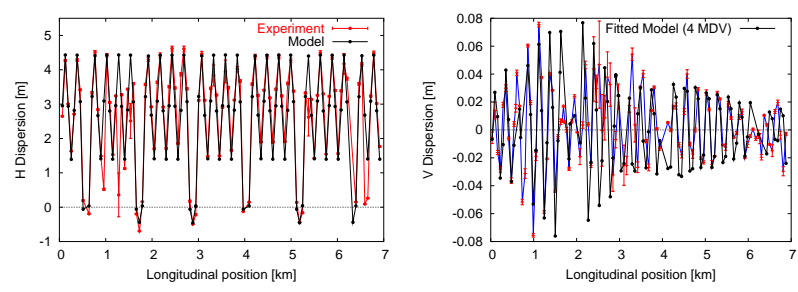

Figure 3: Measured horizontal (left) and vertical dispersion (right) compared with the model prediction for 2003. Four correctors were fitted to reproduce the measured vertical dispersion.

quantity $\beta /<\beta>$, where $<>$ denotes the average over the BPM locations (all with equal design $\beta$ value), i.e., we divide the betatron amplitude at one BPM by the average of the betatron amplitudes over all BPMs. The betatron amplitude is obtained from the tune peak in the Fourier spectrum of the turn-by-turn data. Since we have several data sets, for different values of $\delta$, we can extract the chromatic beta function normalized to the average beta by performing a linear fit of $\beta /<\beta>$ versus $\delta$. The slope of this fit equals $\beta^{\prime} /<\beta>$. Its value at all vertical BPMs is shown in Fig. 4 for the 2003 data. At most BPM locations the beta function has a relative variation of about $\pm 3 \%$ for an energy change of $10^{-3}$. The reason for the discrepancy from the model is not known. The multipole errors and even the lattice sextupoles have little effect on this quantity. The horizontal chromatic beta measurement (not shown) is not reliable, since a large number of horizontal BPM readings were missing.

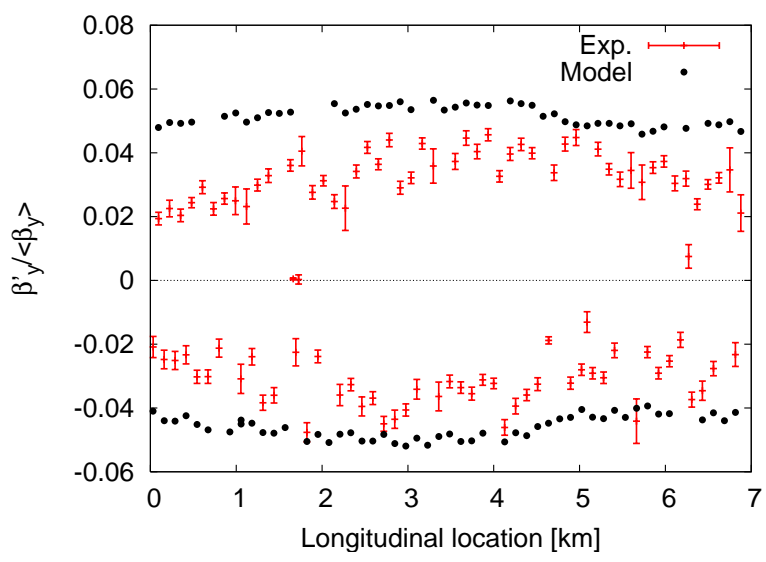

Figure 4: Measured vertical chromatic beta function in 2003 compared with the nonlinear-model prediction.

\section{BPM CALIBRATION}

To verify the absolute scale of the BPM readings, we measured orbit changes while exciting individual correctors. For the horizontal plane the orbit difference between the two following configurations was considered: corrector MDH432 powered at $30 \mu \mathrm{rad}$ and $\mathrm{MDH} 430$ at 30 $\mu \mathrm{rad}$. The agreement for the overall scale is good (factor
$0.96 \pm 0.08$ ); see left picture of Fig. 5. In the vertical plane, we used orbits with MDV433 and MDV431, respectively, powered at $30 \mu \mathrm{rad}$. In this case, the model prediction has to be multiplied by a factor $1.42 \pm 0.06$, in order to reproduce the BPM readings (right picture). The reason for the large scale error in the vertical plane is yet unknown.
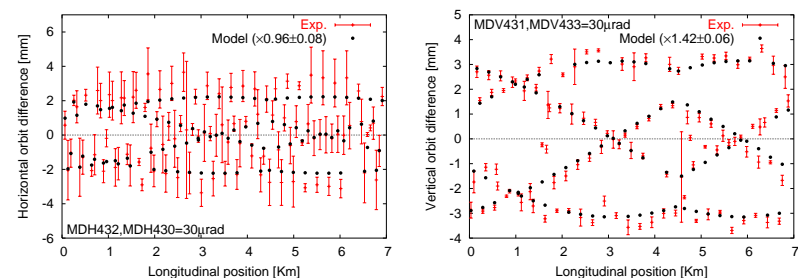

Figure 5: Horizontal orbit difference between powering MDH342 and MDH430 at $30 \mu \mathrm{rad}$ (left); and vertical difference for MDV433 and MDV431 at $30 \mu \mathrm{rad}$; measured in 2003.

\section{RESONANCE DRIVING TERMS}

The BPM data were also analysed to obtain 3rd and 4-th order resonance driving terms $f_{3000}$ and $f_{4000}[8,9]$ at all BPMs around the SPS (see also [2]).

\section{CONCLUSIONS}

In 2003 and 2004, the nonlinear SPS optics model was constructed for two different injection energies and SPS cycles. This model, which was also augmented to include voluntary magnet misalignments, reproduces the measured nonlinear chromaticity over a momentum range of $\pm 0.5 \%$. The horizontal model dispersion is consistent with the direct measurement; the spurious vertical dispersion is small. The measured vertical chromatic beta function seems to be lower than predicted by a factor of about 1.5-2. A similar but opposite factor between measurement and model is found for the vertical BPM calibration using orbit correctors. The central orbit was determined from the variation of the rms horizontal orbit with the average orbit.

\section{REFERENCES}

[1] G. Arduini, et al., CERN SL Note 2001-030 MD; SL Note 2001-049 MD; and Proc. EPAC 2002, Paris, p. 1220.

[2] G. Arduini et al., Proc. PAC2003, Portland, p. 2240; and CERN AB Note 2003-025 (ABP) (2003).

[3] J. Wenninger, CERN SL-Note-2002-041 OP.

[4] G. Arduini, A. Faus-Golfe and R. Tomás, AB Note 2003042 (ABP) (2003).

[5] H. Grote, F.C. Iselin, CERN/SL/90-13(AP)" (1995).

[6] V. Hatton, A. Riche, A. Swift, Lab II/MA-Int. 75-2; M. Cornaccia, Lab II-DI-PA/Int. 75-8 (1975).

[7] F. Schmidt, CERN SL/94-56 (AP).

[8] R. Tomas, PhD thesis, U. de Valencia 2003.

[9] R. Bartolini, F. Schmidt, Part. Acc. 59, p. 93 (1998). 